\title{
ON THE ENTROPY NORM SPACES AND THE HARDY SPACE $\operatorname{Re} H^{1}$
}

\author{
W. C. LANG
}

(Communicated by J. Marshall Ash)

\begin{abstract}
R. Dabrowski introduced certain natural multiplier operators which map from the entropy norm spaces of $B$. Korenblum into the Hardy space $\operatorname{Re} H^{1}$. We show that the images of the entropy norm spaces in $\operatorname{Re} H^{1}$ do not include all of that space.
\end{abstract}

\section{INTRODUCTION}

We consider the entropy norm spaces of Korenblum [4]. He defined an entropy function $\kappa:[0,1] \rightarrow[0,1]$ to be a concave, continuous, increasing function with $\kappa(0)=0$. We denote by $K_{0}$ the set of such functions such that $\kappa^{\prime}(0)=\lim _{x \rightarrow 0^{+}} k(x) / x=\infty$. According to Dabrowski [1] to each $\kappa \in K_{0}$ there is a unique probability measure $\mu=\mu_{\kappa}$ such that

$$
\kappa(x)=\int_{0}^{x} \int_{t}^{1} \frac{d \mu(u)}{u} d t .
$$

Then the entropy norm of a continuous 1-periodic function $f \in C(T)$ (where $T=R \bmod 1)$ is given by

$$
\|f\|_{\kappa}=\int_{0}^{1} \int_{T} \Omega_{I}(f) d t \frac{d \mu(s)}{s}
$$

where $I=[t-s / 2, t+s / 2]$ and where $\Omega_{I}(f)=\sup \{|f(u)-f(v)|: u, v \in I\}$. (This norm was introduced by Korenblum [4]; this formula for the norm is due to Dabrowski [4].) We denote by $C_{\kappa} \subseteq C(T)$ the space of continuous 1 -periodic functions of finite entropy norm.

In [2], Dabrowski introduced an operator $T_{\kappa}: C_{\kappa} \rightarrow \operatorname{Re} H^{1}$, given by

$$
T_{\kappa} f(t)=\int_{T} \int_{0}^{1} \frac{\chi_{I}(t)}{s^{2}}(f(t)-f(I)) d \mu(s) d x
$$

where $I=[x-s / 2, x+s / 2], \quad f(I)=\frac{1}{|I|} \int_{I} f(t) d t$ is the average of $f$ over $I$, and $\chi_{I}$ is the usual characteristic function of $I$. He showed that $T_{\kappa}$ is a

Received by the editors November 6, 1991; presented March 20, 1992 in the Special Session on Harmonic Analysis of the 873rd Meeting of the AMS, at Southwest Missouri State University.

1991 Mathematics Subject Classification. Primary 42A20, 46E15.

Key words and phrases. Entropy norm spaces, real Hardy space. 
multiplier with coefficients

$$
\beta_{n}=\beta_{n}(\kappa)=\frac{1}{2 \pi^{2} n^{2}} \int_{(0,1]}\left(\cos (2 \pi n s)-1+2 \pi^{2} n^{2} s^{2}\right) \frac{1}{s^{3}} d \mu_{\kappa}(s)
$$

(for $n>0$ we set $\beta_{-n}=\beta_{n}$ and $\beta_{0}=0$ ). In [3], Dabrowski asked the question: given $f \in \operatorname{Re} H^{1}$, are there $\kappa \in K_{0}$ and $g \in C_{\kappa}$ such that $f=T_{\kappa} g$ ? (One reason why this question is of interest is because, as Dabrowski remarks, a positive answer would imply the Fefferman duality $\left(\operatorname{Re} H^{1}(0)\right)^{*}=$ BMO.

\section{THE MAIN RESUlT}

We are ready to give a negative answer to this question.

Theorem. There is a function $f \in \operatorname{Re} H^{1}$ such that there are no $\kappa \in K_{0}$ and $g \in C_{\kappa}$ with $f=T_{\kappa} g$.

Proof. We construct $f$ as follows. Let $h$ be the function with Fourier series $\sum_{n=1}^{\infty}(\sqrt{n} \log (n+1))^{-1} e_{n}$, where $e_{n}=e^{2 \pi i n t}$. Then $h \in H^{2}$. So $h^{2} \in H^{1}$ (see, e.g., Zygmund [6, VII (7.22), p. 275]). We let $f=\operatorname{Re}\left(h^{2}\right)$. So of course $f \in \operatorname{Re} H^{1}$. We have

$$
h^{2} \sim \sum_{n=1}^{\infty}\left(\sum_{j=1}^{n-1} b_{j} b_{n-1}\right) e_{n}
$$

where $b_{j}=(\sqrt{j} \log (j+1))^{-1}$. It is not hard to show that $f$ has Fourier series $\sum_{n=1}^{\infty} a_{n} \cos (2 \pi n t)$ where $a_{n} \geq$ const. $(\log (n+1))^{-2}$ for $n=1,2,3, \ldots$.

Now we suppose that there is a $\kappa \in K_{0}$ and a $g \in C_{\kappa}$ such that $T_{\kappa} g=f$. We write $g$ as $\sum c_{n} e_{n}$. Then since $T_{\kappa} g=f$ we have $c_{n}=a_{n} / \beta_{n}, \quad n \geq 1$. This enables us to write $g$ as $\sum_{1}^{\infty} c_{n} \cos (2 \pi n t)$ where $c_{n} \geq 0$ for all $n>0$.

We assume that $g \in C_{\kappa}$ which implies that $g$ is bounded. Consequently (since $g$ has a cosine series with positive coefficients), we must have $\sum c_{n}<\infty$ or $\sum a_{n} / \beta_{n}<\infty$. Therefore

$$
\sum_{n=1}^{\infty}\left(\frac{1}{\log (n+1)}\right)^{2} \frac{1}{\beta_{n}}<\infty
$$

We must also have

$$
\sum_{n=1}^{\infty} \frac{1}{n^{2}} \beta_{n}<\infty
$$

[By Lang [5], $\beta_{n}$ compares with $n \kappa(1 / n)-n^{2} \int_{0}^{1 / n} \kappa(t) d t=\bar{\kappa}^{\prime}(1 / n)$ where $\bar{\kappa}(x)=\frac{1}{x} \int_{0}^{x} \kappa(t) d t$. We have $\bar{\kappa}(x)=\int_{0}^{x} \bar{\kappa}^{\prime}(t) d t$, so this integral must be convergent; we may estimate this integral by the sum

$$
\sum_{n=1}^{\infty}\left(\frac{1}{n}-\frac{1}{n+1}\right) \bar{\kappa}^{\prime}\left(\frac{1}{n}\right) \approx \sum_{n=1}^{\infty} \frac{1}{n^{2}} \beta_{n} .
$$

(Note that $\bar{\kappa}^{\prime}(x)=\left(1 / x^{2}\right)\left(x \kappa(1 / x)-\int_{0}^{x} \kappa(t) d t\right)$ is the product of $1 / x^{2}$ and a function which goes to 0 monotonically as $x \rightarrow 0$. So the integral and the sum compare.)] 
But (1) and (2) are not compatible. Indeed, suppose the sums (1) and (2) are both finite. Then by the Cauchy-Schwarz inequality

$$
\begin{aligned}
\sum_{n=1}^{\infty} \frac{1}{n} \frac{1}{\log (n+1)} & =\sum_{n=1}^{\infty}\left(\frac{1}{n} \sqrt{\beta_{n}}\right)\left(\frac{1}{\log (n+1)} \frac{1}{\sqrt{\beta_{n}}}\right) \\
& \leq\left(\sum_{n=1}^{\infty} \frac{1}{n^{2}} \beta_{n}\right)^{1 / 2}\left(\sum_{n=1}^{\infty}\left(\frac{1}{\log (n+1)}\right)^{2} \frac{1}{\beta_{n}}\right)^{1 / 2}<\infty,
\end{aligned}
$$

which is nonsense. So there cannot be $\kappa \in K_{0}, g \in C_{\kappa}$ such that $T_{\kappa} g=f$, and we are done.

\section{REFERENCES}

1. R. Dabrowski, Probability measure representation of norms associated with the notion of entropy, Proc. Amer. Math. Soc. 90 (1984), 263-268.

2. Bull. Amer. Math. Soc. (N.S.) 18 (1988), 49-51.

3. __ On a natural connection between the entropy spaces and Hardy space $\operatorname{Re} H^{1}$, Proc. Amer. Math. Soc. 104 (1988), 812-818.

4. B. Korenblum, On a class of Banach spaces associated with the notion of entropy, Trans. Amer. Math. Soc. 290 (1985), 527-553.

5. W. C. Lang, A growth condition for Fourier coefficients of a function of bounded entropy norm, Proc. Amer. Math. Soc. 112 (1991), 433-439.

6. A. Zygmund, Trigonometric series, 2nd ed., vol. I, Cambridge Univ. Press, Cambridge, 1959.

Department of Mathematics and Statistics, Mississippi State University, Mississippi STATE, MississiPPI 39762

E-mail address: lang@math.msstate.edu 